\title{
NMR Study of the Time Dependent Dipolar Splitting of Methylene Chloride Protons in Poly( $\gamma$-benzyl L-glutamate)
}

\author{
Yoshio Yokoyama, Makoto Arai, and Atsuo Nishioka \\ Department of Polymer Chemistry, Tokyo Institute of Technology, \\ 12-1, Ookayama, 2-chome, Meguro-ku, Tokyo 152, Japan.
}

(Received November 11, 1976)

\begin{abstract}
Poly( $\gamma$-benzyl L-glutamate) in a concentrated methylene chloride solution is in a liquid crystalline state. The solvent signal of this solution is a doublet due to magnetic dipole-dipole interactions. Separation of the doublet varies with residence time of the sample in the magnetic field after insertion in the NMR probe or after changing the direction of the applied field. This is attributed to the time dependence of the orientation or reorientation of molecules in the magnetic field.

The time dependent phenomena were observed by ${ }^{1} \mathrm{H}-\mathrm{FT}-\mathrm{NMR}$ and a floppy disk drive system. That is, after changing the direction of the applied field the peak height of the upfield one of the methylene chloride doublets first decreases gradually relative to the downfield one, and still later the peak height of the upfield one increases, and finally both peaks become of equal height. This is interpreted through the anisotropy of the diamagnetic susceptibility $\Delta \chi$ for oriented nematic liquid crystals. The theory is given and the potential for obtaining structural information about nematic liquid crystals of polypeptide is discussed.

KEY WORDS NMR / PBLG / Liquid Crystal / Methylene Chloride / Orientation / Nematic / Cholesteric / Polypeptide /
\end{abstract}

Poly( $\gamma$-benzyl L-glutamate) (PBLG) is well known to form lyotropic liquid crystals in various helicogenic solvents, and has an $\alpha$-helix conformation in these solvents.

In the nematic liquid crystalline state, the rod-like molecules are arranged parallel to one another. The packing of rod-like helices appears to be locally hexagonal on a microscopic scale. ${ }^{4}$

Specific solvent-polymer interactions are not responsible for this behavior. Liquid crystal formation is spontaneous when rod-like, $\alpha$-helical polypeptides exceed a critical concentration in solution. Experimental observations are in reasonable agreement with the self-ordering theory, ${ }^{5}$ and show that the critical polypeptide concentration depends primarily on the axial ratio of the rod-like polymer molecules. ${ }^{7}$

When the PBLG molecules in methylene chloride solution forms a liquid crystal, the polymer segments are so restricted that the direct dipole-dipole interactions of protons in the polymer are not averaged out. Therefore, the NMR signals of PBLG become so broad that they can not be observed by the ordinary high resolution NMR technique. However, the solvent signals can still be observed in the liquid crystalline state, though they are much broader than those in isotropic solutions and are split into a doublet. Sobajima ${ }^{1}$ was the first to observe this dipolar splitting. Orwoll and Vold $^{8}$ showed theoretically and experimentally the time dependence of methylene chloride dipolar splitting on the average orientation of the polymer helices with respect to the external field.

It was expected originally that the doublets would have the same peak height and width, but unequal peak heights of the doublets were reported in a previous study, ${ }^{8}$ and a detailed discussion was not given. The doublets show equal peak heights at equilibrium, but transient unequal peak heights were observed in the course of coming back to equilibrium after rotation of 
the nematic director.

It is impossible to give a rigorous discussion about time dependent phenomena obtained from CW-NMR spectra. The purpose of this paper is to report some results of the time dependent reorientation of PBLG liquid crystals after the direction of the applied magnetic field is changed and to clarify the mechanism of the asymmetric line shape of the doublet by ${ }^{1} \mathrm{H}-\mathrm{FT}-\mathrm{NMR}$ online with a floppy disk drive system developed in our laboratory.

\section{THEORY}

When a sample of PBLG in methylene chloride is placed in a magnetic field $H_{0}$, the anisotropy of the diamagnetic susceptibility of the PBLG molecules results in forces which align them parallel to the magnetic field. And when the equilibrated nematic director was rotated mechanically from $H_{0}$, it tended to reorient again to $H_{0}$.

In the course of the reorientation of the liquid crystals, asymmetric line shapes of the doublet of the methylene chloride protons have been observed, depending upon the time after the director was rotated and the rotational angle $\theta$.

The transient discrepancies of the peak heights of the doublets (dowfield peak is higher) were found to change systematically with time and rotational angle $\theta$. We interpret this phenomena to refer only to the anisotropy of the diamagnetic susceptibility of PBLG $\alpha$-helices. This is reasonable since methylene chloride molecules are mobile even in a nematic PBLG liquid crystal and the chemical shift anisotropy was averaged out.

We assume a cylindrical symmetry for the rod-like helical molecules. The magnetic field lies on the $z$-axis and at equilibrium the nematic director coincides with the same axis. The axis of the cylindrical sample tube lies on the $x$-axis.

When the sample tube is rotated about the $x$-axis by an amount $\theta$, the orientation of the nematic director is also changed.

In that situation, the tensor $\chi$ of diamagnetic susceptibility at $\theta$ may be obtained by

$\chi=\left(\begin{array}{ccc}1 & 0 & 0 \\ 0 & \cos \theta & \sin \theta \\ 0 & -\sin \theta & \cos \theta\end{array}\right)\left(\begin{array}{ccc}\chi_{\perp} & 0 & 0 \\ 0 & \chi_{\perp} & 0 \\ 0 & 0 & \chi_{I I}\end{array}\right)\left(\begin{array}{ccc}1 & 0 & 0 \\ 0 & \cos \theta & -\sin \theta \\ 0 & \sin \theta & \cos \theta\end{array}\right)$ $=\left(\begin{array}{ccc}\chi_{\perp} & 0 & 0 \\ 0 & \chi_{\perp} \cos ^{2} \theta+\chi_{\| /} \sin ^{2} \theta & \left(\chi_{\|}-\chi_{\perp}\right) \cos \theta \sin \theta \\ 0 & \left(\chi_{\| /}-\chi_{\perp}\right) \cos \theta \sin \theta & \chi_{\perp} \sin ^{2} \theta+\chi_{\| /} \cos ^{2} \theta\end{array}\right)$

And the induced magnetization becomes

$$
\boldsymbol{M}=\chi \cdot\left(\begin{array}{c}
0 \\
0 \\
H_{0}
\end{array}\right)=\left(\begin{array}{c}
0 \\
\left(\chi_{\| /}-\chi_{\perp}\right) \cos \theta \sin \theta H_{0} \\
\left(\chi_{\perp} \sin ^{2} \theta+\chi_{\| /} \cos ^{2} \theta\right) H_{0}
\end{array}\right) \text {. }
$$

So the $y$ and $z$ axis components of the magnetization are $\left(\chi_{\|}-\chi_{\perp}\right) \cos \theta \sin \theta H_{0} \quad$ and $\left(\chi_{\perp} \sin ^{2} \theta+\chi_{\|} \cos ^{2} \theta\right) H_{0}$ recpectively (see Figure 1$)$.

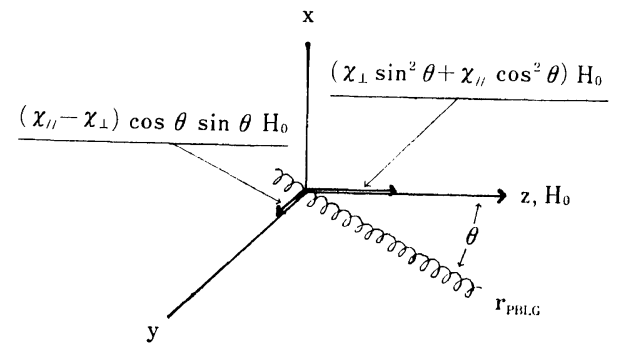

Figure 1. Magnetization induced by the applied magnetic field $H_{0}$. The axis of PBLG rotates by an amount $\theta$ in the $y-z$ plane.

We may neglect the $y$ component, because it is much smaller than the $z$ component. Then the effective field in a cylindrical sample tube along the $z$ axis $\left(H_{\mathrm{i}}\right)$ is given by the following equation:

$$
\begin{aligned}
H_{\mathrm{i}} & =\left(1-\frac{2}{3} \pi \chi\right) H_{0} \\
& =\left\{1-\frac{2}{3} \pi\left(\chi_{\perp} \sin ^{2} \theta+\chi_{\|} \cos ^{2} \theta\right)\right\} H_{0} \\
& =\left[1-\frac{2}{3} \pi\left\{\frac{1}{2}\left(\chi_{\perp}+\chi_{I I}\right)+\frac{1}{2}\left(\chi_{H}-\chi_{\perp}\right) \cos 2 \theta\right\}\right] H_{0}
\end{aligned}
$$

This indicates that the local field in the PBLG liquid crystal varies with $\cos 2 \theta$. The point of inflexion is at $\theta=45$.

The calculated maximum variation of the resonant frequency $\left(C_{0}\right)$ is $6.7 \mathrm{~Hz}$, using the diamagnetic susceptibility obtained by Samulski, et al. ${ }^{9}$ Figure 2 shows the variation of the chemical shifts at the center of the doublets $\left(\nu_{\mathrm{c}}\right)$ and their splitting with $\theta(a, b)$. The splitting is calculated by $S=\frac{1}{2}\left|3 \cos ^{2} \theta-1\right| S_{0}$; here $S_{0}$ is the splitting at equilibrium (in our case $S_{0}=53.2 \mathrm{~Hz}$ ). Below the magic angle, the slope 
Dynamic Phenomena of PBLG Liquid Crystal

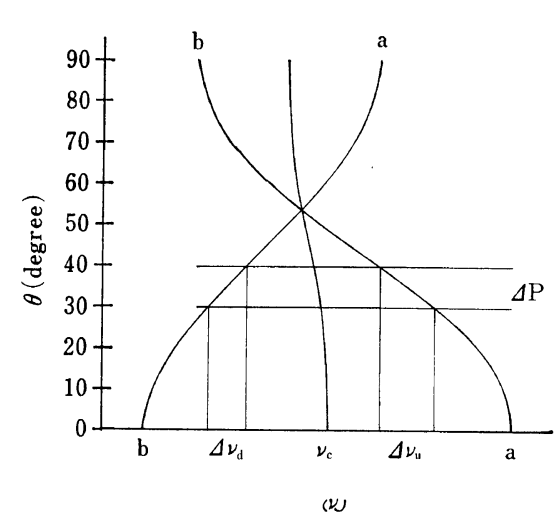

Figure 2. The relationship between the distribution of nematic director $(\Delta P)$ and the distribution of the resonant frequency $\left(\Delta \nu_{\mathrm{d}}\right.$ and $\left.\Delta \nu_{\mathrm{u}}\right)$. The vertical scale indicates the angle of nematic director and the horizontal scale indicates the frequency.

of the upfield chemical shift curve (a) is smaller than that of the downfield chemical shift curve (b), because the center of the doublets shifts downfield with $\cos 2 \theta$.

If the orientation of the PBLG $\alpha$-helices distributes in an angle $\Delta P$, the resonant frequency of the upfield peak spreads in a range $\Delta \nu_{u}$, and the downfield peak spreads in $\Delta \nu_{\mathrm{d}}$. The figure shows clearly that $\Delta \nu_{\mathrm{u}}$ is greater than $\Delta \nu_{\mathrm{d}}$, so the half height width of the upfield peak becomes broader than that of the downfield peak.

Now let us consider the location of the point of maximum discrepancy.

$\Delta \nu_{\mathrm{d}}$ and $\Delta \nu_{\mathrm{u}}$ are given by the following equation below the magic angle (see Figure 2). Above the magic angle, $\Delta \nu_{\mathrm{d}}$ and $\Delta \nu_{\mathrm{u}}$ must be exchanged.

$$
\begin{aligned}
\Delta \nu_{\mathrm{d}}= & {\left[\nu_{\mathrm{c}}-\frac{S_{0}}{4}\left\{3 \cos ^{2}\left(\theta+\frac{1}{2} \Delta P\right)-1\right\}\right.} \\
& \left.-\frac{C_{0}}{2} \cos 2\left(\theta+\frac{1}{2} \Delta P\right)\right] \\
& -\left[\nu_{\mathrm{c}}-\frac{S_{0}}{4}\left\{3 \cos ^{2}\left(\theta+\frac{1}{2} \Delta P\right)-1\right\}\right. \\
& \left.-\frac{C_{0}}{2} \cos 2\left(\theta-\frac{1}{2} \Delta P\right)\right] \\
= & \frac{3}{4} S_{0}\left\{\cos ^{2}\left(\theta-\frac{1}{2} \Delta P\right)\right. \\
& \left.-\cos ^{2}\left(\theta+\frac{1}{2} \Delta P\right)\right\}
\end{aligned}
$$

$$
\begin{aligned}
& -\frac{C_{0}}{2}\left\{\cos 2\left(\theta-\frac{1}{2} \Delta P\right)\right. \\
& \left.-\cos 2\left(\theta+\frac{1}{2} \Delta P\right)\right\} \mid \\
= & \left|\left(\frac{3}{4} S_{0}-C_{0}\right) f(\theta)\right|
\end{aligned}
$$

here

$$
f(\theta)=\cos ^{2}\left(\theta-\frac{1}{2} \Delta P\right)-\cos ^{2}\left(\theta+\frac{1}{2} \Delta P\right) .
$$

Similarly,

$$
\begin{aligned}
\Delta \nu_{\mathrm{u}}= & {\left[\nu_{\mathrm{c}}+\frac{S_{0}}{4}\left\{3 \cos ^{2}\left(\theta-\frac{1}{2} \Delta P\right)-1\right\}\right.} \\
& \left.+\frac{C_{0}}{2} \cos 2\left(\theta-\frac{1}{2} \Delta P\right)\right] \\
& -\left[\nu_{\mathrm{c}}+\frac{S_{0}}{4}\left\{3 \cos ^{2}\left(\theta+\frac{1}{2} \Delta P\right)-1\right\}\right. \\
& \left.+\frac{C_{0}}{2} \cos 2\left(\theta-\frac{1}{2} \Delta P\right)\right] \\
= & \left|\left(\frac{3}{4} S_{0}+C_{0}\right) f(\theta)\right|
\end{aligned}
$$

where the term of $\cos 2 \theta$ is derived from the theory mentioned above. If the total peak areas and the ratios of the downfield peak area to the upfield one are constant throughout an experiment, the ratio of the relative peak heights $(R)$ is given by the following equation, assuming an isosceles triangle for the shape of a peak:

$$
R=\frac{H_{\mathrm{d}}}{H_{\mathrm{u}}}=\frac{\Delta \nu_{\mathrm{u}}+\Delta \nu_{1 / 2}}{\Delta \nu_{\mathrm{d}}+\Delta \nu_{1 / 2}}
$$

where $H_{\mathrm{d}}$ and $H_{\mathrm{u}}$ are the peak heights of the downfield peak and upfield one respectively. $\Delta \nu_{1 / 2}$ is the half height width at thermal equilibrium.

Equation 6 has a maximum at $45^{\circ}$, the maximum value 1.2 is obtained using the values of $S_{0}=53.2 \mathrm{~Hz}, C_{0}=6.7 \mathrm{~Hz}, \Delta \nu_{1 / 2}=6.0 \mathrm{~Hz}$, and $\triangle P=10^{\circ}$.

Thus, the maximum discrepancy of the relative peak heights occurs at $45^{\circ}$, even if the distribution of the orientational axes of PBLG molecules is fixed.

The more the rotational angle of the director increased, the broader becomes the distribution of the orientational axes of PBLG molecules. Therefore, it is anticipated that there will be a larger discrepancy in the relative peak heights 


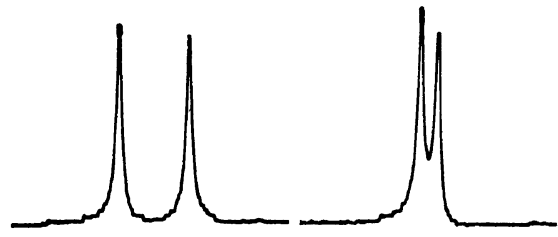

$\infty$

0

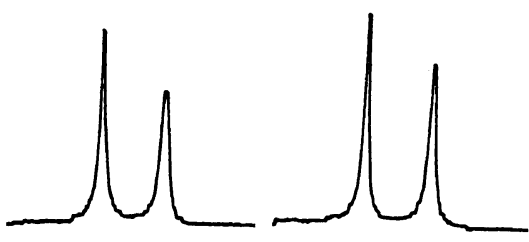

30

42

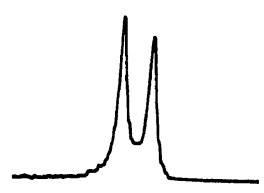

6

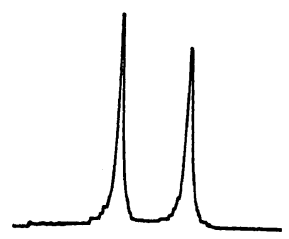

54

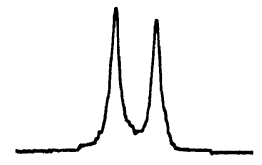

12

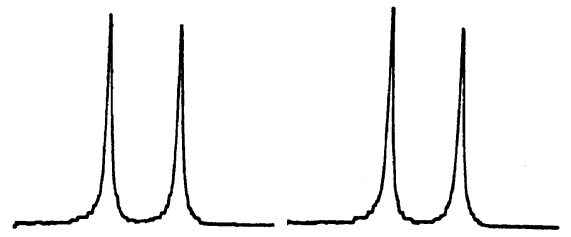

192

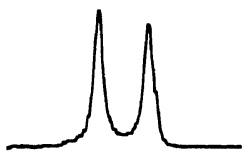

18

438

Figure 3. The NMR spectra observed after an equilibrium sample was rotated to $50^{\circ}$. The numbers under the spectra give the number of seconds after the rotation.

as the rotational angle increases.

\section{EXPERIMENTAL}

The proton NMR spectra of methylene chloride were obtained at room temperature $\left(25 \pm 1^{\circ} \mathrm{C}\right)$ using a JEOL's PS-100 FT-NMR spectrometer equipped with a floppy disk drive $(F D)^{10}$. This FT-NMR-FD system was developed to catch moderately rapid kinetic phenomena by fast transfer of successive free induction decay signals to a floppy disk. The system can acquire NMR spectra every $2.5 \mathrm{sec}$ at maximum.

PBLG of molecular weight 75,000 was purchased from Miles-Yeda, Ltd., and spectrograde methylene chloride was purchased from Tokyo Kasei Kogyo, Ltd. Neither reagent was further purified. The samples were sealed in standard 5-mm NMR tubes without degassing; polymer concentrations were adjusted to $20 \mathrm{wt} \%$. The homogeneity of viscous samples was ensured by inverting the sample tube many times prior to placement in the magnetic field for about $4 \mathrm{hr}$. After adjusting the field to the best homogeneity, the liquid crystal sample was placed in the field and held to attain equilibrium nematic orientation for about $8 \mathrm{hr}$. The field homogeneity was checked at the onset of every experimental series by observing the methylene chloride signal at equilibrium.
After the sample had reached equilibrium with the field, the NMR tube containing the liquid crystal was rotated by an amount $\theta$, and the change of the spectra was subsequently monitored as a function of time.

Sample tube rotation angles were measured with a calibrated disk placed on the spinner assembly of the probe, with an accuracy of $\pm 2^{\circ}$.

The proton spin-lattice relaxation time $\left(T_{1}\right)$ of methylene chloride in a liquid crystal was $1.4 \mathrm{sec}$, so the interval in a pulse train was set to $6 \mathrm{sec}$, which was 4 times the $T_{1}$ value.

\section{RESULTS AND DISCUSSION}

When the nematic director was changed from the equilibrium direction, the anisotropy of the diamagnetic susceptibility of the PBLG molecules results in forces which realign the PBLG molecules parallel to the magnetic field. Equilibrium is attained by a rotation less than $90^{\circ}$.

Figure 3 shows the measured dipolar splittings as a function of time after rotation of the nematic director. They are more reliable than other observations of the time dependencies made by $\mathrm{CW}-\mathrm{NMR},{ }^{8}$ because the spectra were observed by FT-NMR. The relative peak area of the doublets and the total peak area of the doublets were found to be equal throughout 
these experiments within an experimental error of $\pm 4 \%$.

Figure 4 shows the time dependencies of the relative peak heights which were given as the ratio of the downfield peak height to the upfield one. The ratios depend both on time and the rotational angle, showing maxima at about $30 \mathrm{sec}$ in each case and at about $50^{\circ}$. Figure 5 shows the maximum discrepancies of the peak heights of the doublets $v s$. rotational angle, which are exhibited in the course of coming back to equilibrium.

Under certain circumstances the theory has been found to be valid till $50^{\circ}$, but above this angle reorientation proceeds more rapidly than below this angle and the doublets change in a complex manner. Apparently some changes in the distribution of the nematic director may occur above a critical angle with the field, possibly due to counterrotation of the polypeptide helices.

If the directions of the PBLG axes have the same distribution, the peak height discrepancy became maximum if the nematic director was rotated to an amount $45^{\circ}$ since the distribution of the resonant frequencies became maximum at this angle (see Figure 2).

In our experiment, the maximum discrepancies in the transient spectrum occurred at $50^{\circ}$. This

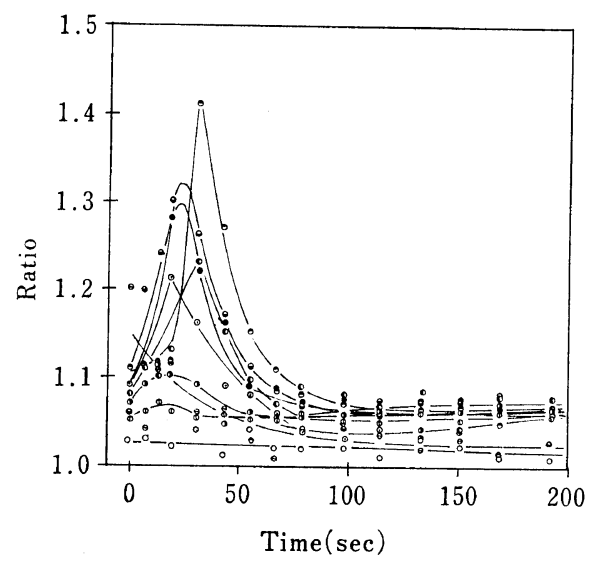

Figure 4. Relative peak heights vs. time. Rotational angles of the nematic director are indicated as: ○, $10^{\circ} ;$ (1), $20^{\circ} ;$ (I, $30^{\circ} ;$ (, $40 ; \ominus, 50^{\circ} ; \ominus, 60^{\circ}$; , $70^{\circ} ; \odot, 80^{\circ} ; \ominus, 90^{\circ}$ respectively. The vertical scale shows relative peak heights of the doublets.

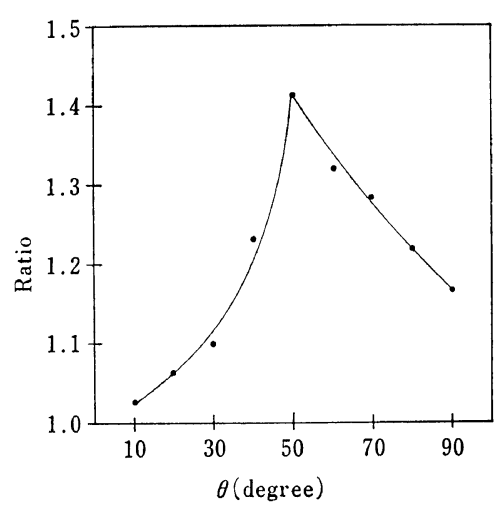

Figure 5. The maximum discrepancies of the peak heights which are exhibited in the course of coming back to equilibrium. The vertical scale is the same as in Figure 4 and the horizontal scale is the rotational angle of the sample tube.

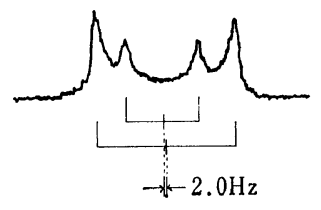

Fignre 6. The NMR spectrum observed after the equilibrated sample was rotated to $90^{\circ}$ for $30 \mathrm{sec}$ and then rotated $-45^{\circ}$.

is interpreted to mean that the distribution of the axes of $\alpha$-helices increases with time after the rotation of the nematic director, and that the nematic director crosses the point of inflexion $\left(45^{\circ}\right)$ of the chemical shift of the doublets. As the theory suggests, the phenomena that show the time dependence in the relative peak heights were illustrated by the superimposition of many doublets.

Further experiments were made to ascertain the chemical shift change due to the orientation of $\alpha$-helices to the applied field. Figure 6 shows the spectrum measured by the method of Orwoll and Vold. $^{8}$ The PBLG of $M_{w} 75,000$ did not show such a spectrum with 4 peaks. It seems that the reorientation rate is so fast that we can not catch such phenomena. So the spectrum in Figure 6 was obtained using a sample of PBDG $\left(M_{w} 300,000 ; \mathrm{CH}_{2} \mathrm{Cl}_{2} 20 \%\right)$. The chemical shift of the center of the outer doublets is located at a higher field than that of the inner doublets by $c a .2 .0 \mathrm{~Hz}$. This is in close 
agreement with our theory, taking into account the distribution of the direction of $\alpha$-helices. If the relative magnitudes of the diamagnetic susceptibilities changed, the exchange of the trend mentioned above can be expected.

The anisotropy of the diamagnetic susceptibility of the PBLG molecules depends mainly on the benzene ring, ${ }^{9,11}$ so it will be possible to discuss the benzene ring orientation to the $\alpha$-helices from the relative peak heights of the doublets.

\section{CONCLUSIONS}

Reorientation processes of PBLG $\alpha$-helices after rotating the nematic director in a magnetic field were observed precisely by ${ }^{1} \mathrm{H}-\mathrm{FT}$-NMRFD system. And the transient unequal peak heights of the methylene chloride doublets were observed.

The phenomena were illustrated quantitatively by two facts. i) The nematic director has a distribution near $\theta$. ii) The chemical shift of the center of the doublets varies with $\theta$ from the change of $\chi$. The unequal peak height results from the superimposition of many doublets; its center varies with $\cos 2 \theta$ and the splitting varies with $\frac{1}{2}\left|3 \cos ^{2} \theta-1\right|$.

Acknowledgment. The authors wish to express their sincere gratitude to Assistant professor $\mathrm{H}$. Tanaka and Mr. T. Hirai for their stimulating discussions and for supplying the sample of PBDG $M_{w} 300,000$. They are also indebted to $\mathrm{Mr}$. H. Tabata for his assistance.

\section{REFERENCES}

1. S. Sobajima, J. Phys. Soc. Jpn., 23, 1070 (1967).

2. E. Iizuka and Y. Go, ibid., 31, 1205 (1971).

3. M. Panar and W. D. Phillips, J. Am. Chem. Soc., 90, 3880 (1968).

4. C. Robinson, J. C. Ward, and R. B. Beevers, Disk. Faraday Soc., 25, 29 (1958),

E. T. Samulski and A. V. Tobolsky, Mol. Cryst. Liq. Cryst., 7, 433 (1969).

5. L. Onsager, Ann. N. Y. Acad. Sci., 56, 627 (1949).

P. J. Flory, Proc. Roy. Soc., Ser. A, 243, 73 (1956).

R. Zwanzig, J. Chem. Phys., 39, 1714 (1963).

E. A. Dimarzio, ibid., 35, 658 (1961).

M. A. Cotter and D. E. Martire, ibid., 52, 1907 (1970).

6. J. J. Hermans, J. Colloid Sci., 17, 638 (1962).

7. E. L. Wee and W. G. Miller, J. Phys. Chem., 75, 1446 (1971).

8. R. D. Orwoll and R. L. Vold, J. Am. Chem. Soc., 93, 5335 (1971).

9. C. G. Sridhar, W. A. Hines, and E. T. Samu1ski, J. Chem. Phys., 61, 947 (1974).

10. Y. Yokoyama, M. Arai, and A. Nishioka, Bunseki Kagaku (Japan Analyst), 26, No. 5 (1977).

11. K. Tohyama and N. Miyata, J. Phys. Soc. Jpn., 34, 1699 (1973). 